\title{
A Teenager of Myelodysplastic Syndrome with Fibrosis and Monosomy 7
}

\author{
Samra Waheed ${ }^{1, *}$, Aisha Jamal ${ }^{2}$, Naeem Abbas², Nida Anwar ${ }^{2}$, Tahir Sultan Shamsi² \\ ${ }^{1}$ Department of Hematology National Institute of Blood Disease \& Bone Marrow Transplantation, Karachi, Pakistan. \\ ${ }^{2}$ Department of Clinical Hematology, National Institute of Blood Disease \& Bone Marrow Transplantation, Karachi, Pakistan.
}

\begin{abstract}
Extensive fibrosis in myelodysplastic syndromes (MDS) is relatively infrequent and associated with worst prognosis. It hasbeen classified as a specific entity by WHO. Moreover, the incidence and prevalence of Myelodyplastic Syndromes in children and adolescents is very low. Herein, we report a case of a 17 years old boy diagnosed as myelodysplastic syndrome with fibrosis along with ofmonosomy 7 . Data pertaining relationship between myelodysplastic syndrome with fibrosis and karyotype abnormalities is relatively sparse. This case report will assist in considering better risk adapted therapies.
\end{abstract}

Keywords: Myelodysplastic syndrome with fibrosis, Monosomy 7, Immunohistochemistry, Fluorescence in situ Hybridization (FISH), Acute myeloid leukemia, HIV.

\section{INTRODUCTION}

Myelodysplastic syndromes (MDS) are composite groups of clonal disorders represented by peripheral blood cytopenias consequential to ineffective and dysplastic hematopoiesis with predisposition to evolve into acute myeloid leukemia (AML) [1] in upto $30 \%$ of patients [2]. In contrast to adulthood, MDS in childhoodis seldom seen, constituting $4 \%$ of all hematological malignancies with an incidence of 1.8 per one million children per year in the $0-14$ year age group [3]. In young adults, it is also rarely reported with worse prognostic implicationand majority of the cases being associated with pancytopenia instead of only anemia as in adults. Most of the time, myelodysplastic syndromes are associated with hypercellular bone marrow but in young adolescents, majority of the cases are reported as hypocellular [4].

Myelofibrosis, on the other hand is bone marrow disorder in which the marrow is replaced by fibrous scar tissue. Numerous benign diseases are related to this condition such as infections including HIV and tuberculosis, osteopetrosis, primary and secondary hyperparathyroidism, autoimmune disorders and certain drugs. Many oncological conditions are also strongly associated with marrow fibrosis such as primary myelofibrosis, post polycythemia or essential thrombocythemia myelofibrosis, chronic myelogenous leukemia, chronic myelomonocytic leukemia, acute leukemia, hairy cell leukemia and metastatic carcinomas [5]. Myelofibrosis is generally related with defects in function or number of platelets and megakaryocytes .

Hyperfibrotic MDS or MDS with myelofibrosis is rare [6] and is about $10-15 \%$ of all MDS, and are categorized as MDS with fibrosis according to WHO classification of haematopoetic

*Address correspondence to this author at the Department of Hematology National Institute of Blood Disease \& Bone Marrow Transplantation, Karachi, Pakistan. E-mail: samraw2010@gmail.com and lymphoid neoplasms 2017 (Revised). These cases usually have advancing clinical course independent of percentage of blast counts.The morphological knowledge from bone marrow aspiration is mostly insufficient and is complemented by histological data and immunohistochemical staining [7]. Unlike Myeloproliferative neoplasms, MDS with fibrosis is not usually associated with splenomegaly and leucoerythroblastosis and usually has dysplastic megakaryocytic morphology especially micromegakaryocytes [8].

\section{CASE PRESENTATION}

We, herein, describe a case of 17 years old boy with no significant past medical or surgical history, presentedwith generalized weakness, dizziness, shortness of breath and fever for 2 -3 months. Examination revealed no visceromegaly or lymphadenopathy. Initial workup was done that showed pancytopenia on complete blood count with $\mathrm{Hb}$ of $8.1 \mathrm{~g} / \mathrm{dl}, \mathrm{MCV}$ of 95.5 $\mathrm{fl}, \mathrm{MCH}$ of $30.7 \mathrm{pg}$ and $\mathrm{MCHC} 32.1 \mathrm{~g} / \mathrm{dl}$. White blood cell count (WBC) was $3.9 \times 10^{9} / \mathrm{L}$ with ANC of $1.5 \times 10^{9} / \mathrm{L}$ and platelet count of $21 \times 10^{9} / \mathrm{L}$. Peripheral film showed dysplastic neutrophils with $03 \%$ blast cells and marked platelet anisocytosis as shown in Figs. $(\mathbf{1}, \mathbf{2})$. Biochemical studies were normal, viral serology was negative. Ultrasound abdomen showed no abnormal findings. Bone marrow aspirate and trephine biopsy was performed. Aspirate washemodiluted (Fig. 3) with occasional blast cells. Trephine biopsy was cellular ( $80 \%$ cellularity) with marked megakaryocytic hyperplasia and features of dysmegakayopoesis, like monolobation and hypolobation. Micromegakaryocytes were also identified on trephine biopsy sections (Fig. 4).Trephine biopsy also revealed markedly increased fibrotic activity that was causing streaming of cells and distortion of morphology. Reticulin stain showed MF-2 fibrosis (Fig. 5). Immunohistochemistry was performed that showed CD34 positivity in around 3\% of cells while CD61 was positive in megakaryocytes and micromegakaryocytes (Fig. 6). Sample for bone marrow karyotyping was taken twice but couldnt be performed due to 
culture failure. On FISH analysis, monosmy 7 was identified in $18 \%$ of the cells. Deletion $5 \mathrm{q}$ was also sent which was negative. According to IPSS-R, our patient was high risk. Such patients have median overall survival of around 1.6 years and increased chance to convert to AML.

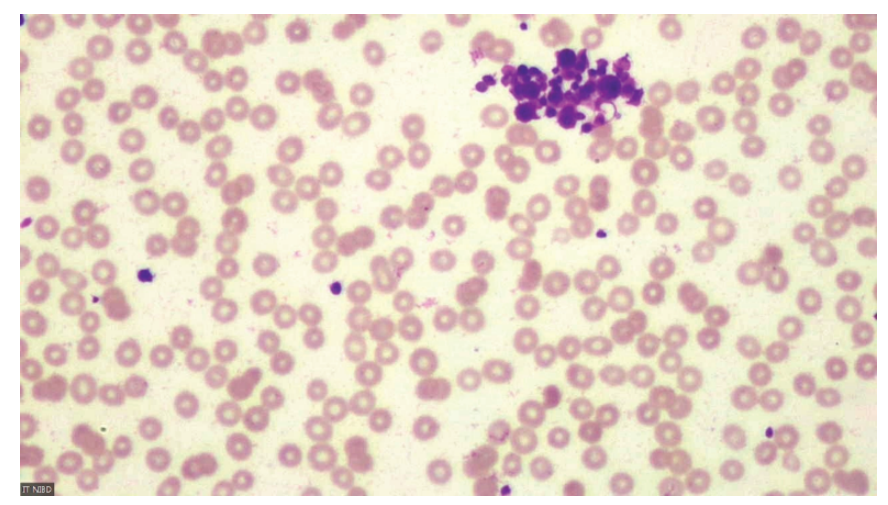

Fig. (1). Peripheral Film Showing Platelet Anisocytosis and Clumping.

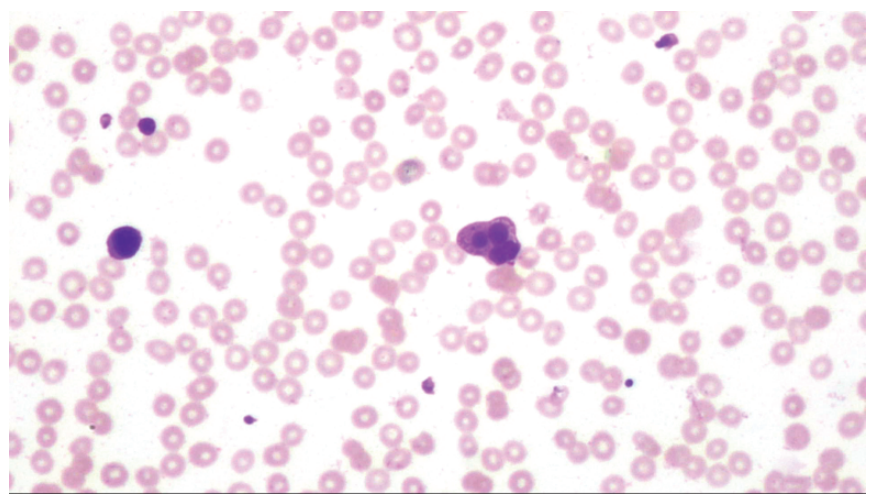

Fig. (2). Peripheral Film Showing Dysplastic Neutrophil.

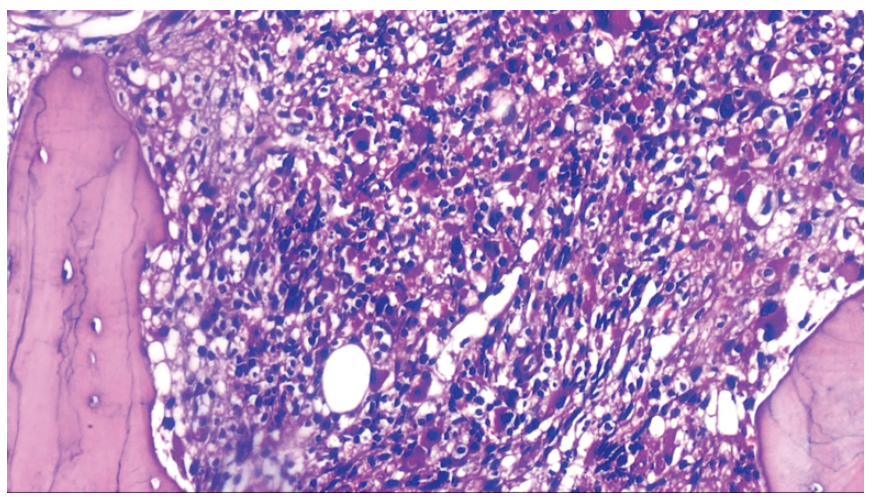

Fig. (3). Trephine Biopsy Section Exhibiting Megakaryocytic Proliferation and Dysplasia.

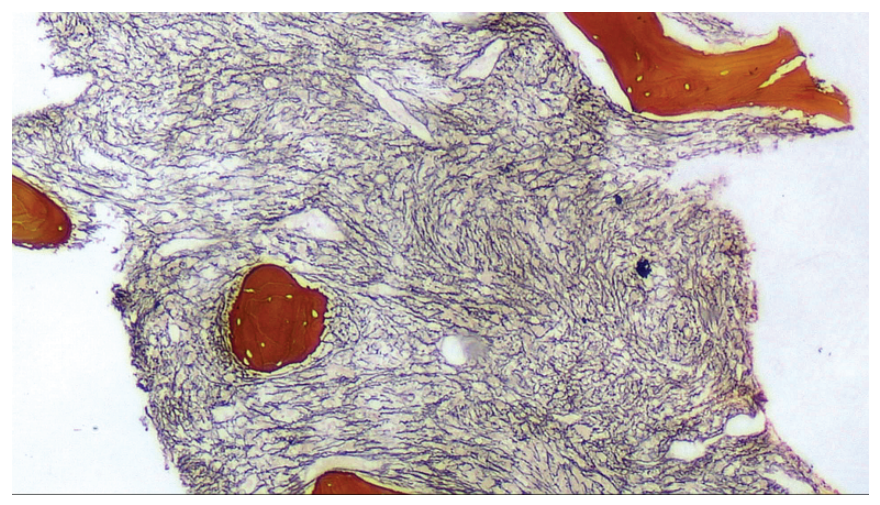

Fig. (4). Reticulin Stain Showing Extensive Fibrosis.

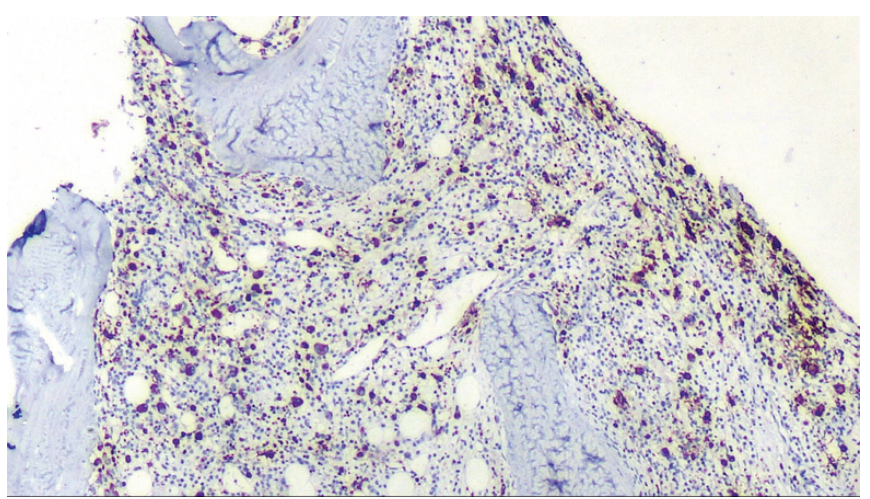

Fig. (5). CD61 Immunohistochemistry on Trephine Biopsy Section.

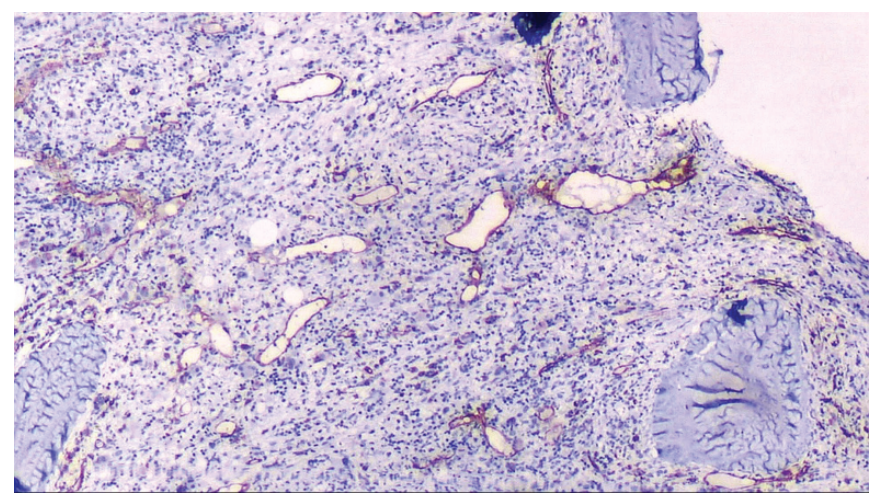

Fig. (6). CD34 Immunohistochemistry on Trephine Biopsy Section.

At present, our patient is planned for treatment by hypomethylating agents followed by bone marrow transplant by HLA matched donor. Good supportive care in the form ofblood product transfusion and antibiotic prophylaxis is also being instituted.

\section{DISCUSSION}

Myelodysplastic syndrome is a rare entity and is generally considered as a disease of elderly. Though uncommon in young patients, MDS has been affiliated with overall poor 
prognosis and decreased overall survival rates. Myelofibrosis (reticulin/collagen) can occur in huge variety of malignant and non-malignant conditions of the bone marrow, including MDS [9]. Patients having MDS-F (Myelodysplastic syndrome with fibrosis) has generally poorer outcome than those having little or no fibrosis $[10,11]$; however, this is also questionable and requires more data analysis $[12,13]$. A large retrospective study of 352 MDS patients showed a median survival of 9.6 months in patients with fibrosis and 17.4 months in those without fibrosis [10]. Reticulin fibrosis is often revocable after therapy however, collagen fibrosis is less likely to be reshaped by treatment. Classical data implied that bone marrow fibrosis modify hematopoietic reconstitution after allogeneic stem cell transplantation. Increased graft failure, delayed neutrophil engraftment and increased risk of relapse were all associated with fibrosis after allogenic stem cell transplantation [14].

Karyotypic abnormalities have been associated with diagnostic and prognostic significance of Myelodysplastic syndrome from previous times. Globally, less than $50 \%$ of MDS patients display an aberrant karyotype, of which del (5q) is the most common cytogenetic abnormality displaying in 10 to $20 \%$ of adult MDS patients [15]. However, loss of chromosome 7 is the most common cytogenetic aberration in pediatric population accounting for around $10-15 \%$ of cases. MDS with monosomy 7 has poor outcome, while isolated del (7q) has a better prognosis comparatively [16]. Monosomy 7 has also been associated with familial myelodysplastic syndrome and GATA 2 mutations and has high propensity to transform to AML. Such patients mostly carry ASXL-1 mutations [17]. Therefore, in young patients, genetic analysis should be performed in routine in order to diagnose, prognosticate and plan treatment accordingly. Myelodysplastic syndrome with fibrosis has not been linked with any specific cytogenetic abnormality though some case reports mention different cytogenetic aberrations associated with poorer outcomes.

22 patients with myeloid malignancies and isochromosome $17 \mathrm{q}$ were associated with myelodysplastic and myeloproliferative features, including pseudo-Pelger-Huet-anamolies,micromegakaryocytes, hypercellularity, fibrosis, and osteosclerosis [18]. This study concluded that disorders with isolated isochromosome $17 \mathrm{q}$ represent a specific entity having myelodysplastic and myeloproliferative features, with increased chances of transformation to leukemia and presence of wild-type TP53 [19]. Though deletion 17p is much commoner in acute myeloid leukemia and myelodysplastic syndrome, isolated deletion $17 \mathrm{q}$ is a rare abnormality.

Treatment of such patients should be aggressive and majority of the studies emphasizeon upfront allogenic HLA matched stem cell transplantation which is the only alleviative option and is associated with better overall and disease free survival. To the best of our literature search, this is the first case of its kind reporting the presence of Myelodysplastic syndrome along with fibrosis and presence of monosmy 7 .

\section{FUNDING}

No funding has been associated with the article.

\section{CONFLICT OF INTEREST}

Declared none.

\section{ACKNOWLEDGEMENTS}

We acknowledge all our fellow colleagues.

\section{REFERENCES}

[1] Heaney M, Golde D. Myelodysplasia. New Engl J Med 1999; 340(21): 1649-60. DOI: 10.1056/NEJM199905273402107

[2] Lambertenghi-Deliliers G, Annaloro C, Oriani A, Soligo D. Myelodysplastic syndrome associated with bone marrow fibrosis. Leuk Lymphoma 1992; 8: 51-5. DOI: $10.3109 / 10428199209049817$

[3] Hasle H, Niemeyer CM, Chessells JM. A pediatric approach to the WHO classification of myelodysplastic and myeloproliferative diseases. Leukemia 2003;17(2): 277-82.

DOI: $10.1038 /$ sj.leu.2402765

[4] Vardiman JW, Thiele J, Arber DA, et al.The 2008 revision of the World Health Organization (WHO) classification of myeloid neoplasms and acute leukemia: Rationale and important changes. Blood 2009; 1145(5): 937-51.

DOI: 10.1182/blood-2009-03-209262

[5] Tefferi A. Pathogenesis of myelofibrosis with myeloid metaplasia. J Clin Oncol 2005; 23(33): 8520-30.

DOI: $10.1200 /$ JCO.2004.00.9316

[6] Das P, Deepali J, Das R, Garewal G. Hyperfibrotic myelodysplastic syndrome: A report of three cases from north India. Turk J Hematol 2009; 26: 93-6.

[7] Bowen D, Culligan D, Jowitt S, et al. Guidelines for the diagnosis and therapy of adult myelodysplastic syndromes. $\mathrm{Br}$ J Haematol 2003; 120(2): 187-200.

DOI: $10.1046 /$ j.1365-2141.2003.03907.x

[8] Rosati S, Anastasi J, Vardiman J. Recurring diagnostic problems in the pathology of the myelodysplastic syndromes. Semin Hematol 1996; 33: 111-26.

[9] Steensma DP, Hanson CA, Letendre L, Tefferi A. Myelodysplasia with fibrosis: A distinct entity? Leuk Res 2001; 25(10): 829-38. DOI: 10.1016/S0145-2126(01)00055-8

[10] Maschek H, Georgii A, Kaloutsi V, et al. Myelofibrosis in primary myelodysplastic syndromes: A retrospective study of 352 patients. Eur J Haematol 1992; 48(4): 208-14.

DOI: 10.1111/j.1600-0609.1992.tb01587.x 
[11] Cunningham SJ, MacCallum MD, NichollsBK, et al. The myelodysplastic syndromes: An analysis of prognostic factors in 226 cases from a single institution. Br J Haematol 1995; 90(3): 602-6. DOI: 10.1111/j.1365-2141.1995.tb05590.x

[12] Rios A,Canizo MC, Sanz MA, et al. Bone marrow biopsy in myelodysplastic syndromes: Morphological characteristics and contribution to the study of prognostic factors. $\mathrm{Br} \mathrm{J}$ Haematol 1990; 75(1): 26-33.

DOI: $10.1111 / \mathrm{j} .1365-2141.1990 . t b 02612 . \mathrm{x}$

[13] Verhoef GE, De Wolf-Peeters C, Ferrant A, et al. Myelodysplastic syndromes with bone marrow fibrosis: A myelodysplastic disorder with proliferative features. Ann Hematol 1991; 63: 235-41. DOI: 10.1007/BF01698371

[14] Della Porta MG, Malcovati L. Myelodysplastic syndromes with bone marrow fibrosis. Haematologica 2010; 96(2): 180-3. DOI: 10.3324/haematol.2010.039875

[15] Solè F, Lu-o E, Sanzo C, et al. Identification of novel cytogenetic markers with prognostic significance in a series of 968 patients with primary myelodysplastic syndromes. Haematologica 2005; 90: 1168-78.
[16] Brandwein JM, Horsman D, Eaves A, et al. Childhood myelodysplasia: Suggested classification as myelodysplastic syndromes. Am J Pediatr Hematol Oncol 1990; 12: 63.

DOI: $10.1097 / 00043426-199021000-00012$

[17] Keel SB, Scott A, Sanchez-Bonilla M, et al. Genetic features of myelodysplastic syndrome and aplastic anemia in pediatric and young adult patients. Haematologica 2016; 101(11): 1343-50. DOI: 10.3324/haematol.2016.149476

[18] Kanagal-Shamanna R, Bueso-Ramos CE, Barkoh B, et al. Myeloid neoplasms with isolated isochromosome $17 \mathrm{q}$ represent a clinicopathologic entity associated with myelodysplastic/myeloproliferative features, a high risk of leukemic transformation, and wild-type TP53. Cancer 2012; 118: 2879-88. DOI: $10.1002 /$ encr.26537

[19] Grimwade D, Hills RK, Moorman AV, et al. Refinement of cytogenetic classification in acute myeloid leukemia: Determination of prognostic significance of rare recurring chromosomal abnormalities among 5876 younger adult patients treated in the United Kingdom medical research council trials. Blood 2010; 116: 354-65.

DOI: $10.1182 /$ blood-2009-11-254441 